\title{
Erratum to: High Content Screening
}

\section{Paul A. Johnston and Oscar J. Trask}

\section{Erratum to:}

Paul A. Johnston and Oscar J. Trask (eds), High Content Screening: A Powerful Approach to Systems Cell Biology and Phenotypic Drug Discovery, Methods in Molecular Biology, vol. 1683

DOI 10.1007/978-1-4939-7357-6

The affiliation of one of the volume editors, Paul A. Johnston, was incorrect in the originally published volume. It has been revised in the current version of the book to reflect the actual affiliation:

Department of Pharmaceutical Sciences, School of Pharmacy, University of Pittsburgh, Pittsburgh, PA, USA

The volume frontmatter reflects this change, as well as the online metadata. 